\title{
Higgs boson pair production at the LHC: top-quark mass effects at next-to-leading order
}

\author{
Jonathan Grigo \\ Karlsruhe Institute of Technology (KIT), Karlsruhe, Germany \\ E-mail: jonathan.grigo@kit.edu \\ Jens Hoff \\ Karlsruhe Institute of Technology (KIT), Karlsruhe, Germany \\ E-mail: jens.hoff@kit.edu

\section{Kirill Melnikov} \\ Johns Hopkins University, Baltimore, MD, USA \\ E-mail: melnikov@pha.jhu.edu

\section{Matthias Steinhauser*} \\ Karlsruhe Institute of Technology (KIT), Karlsruhe, Germany \\ E-mail: matthias.steinhauser@kit.edu
}

\begin{abstract}
Higgs boson pair production is considered at next-to-leading order with special emphasis on the effect of a finite top quark mass. It is shown that, unlike for single-Higgs boson production, power-suppressed corrections are numerically important.
\end{abstract}

11th International Symposium on Radiative Corrections (Applications of Quantum Field Theory to Phenomenology)

22-27 September 2013

Lumley Castle Hotel, Durham, UK

${ }^{*}$ Speaker. 


\section{Introduction}

Since the discovery of a Higgs boson at the CERN Large Hadron Collider (LHC) $[1,2]$ there has been an enormous activity aiming for the determination of the properties of the new particle. Among them are the spin, the couplings to fermions and bosons and the self-coupling. The latter is part of the Higgs boson potential which can be written as

$$
V_{\mathrm{H}}=\frac{1}{2} m_{H}^{2} H^{2}+\lambda v H^{3}+\frac{1}{4} \lambda H^{4},
$$

where $H$ is the physical Higgs boson field and $v$ is the vacuum expectation value. In the Standard Model (SM) we have $\lambda^{\mathrm{SM}}=m_{H}^{2} /\left(2 v^{2}\right) \approx 0.13$. A promising candidate for an observable which is sensitive to $\lambda$ is double-Higgs boson production. It has the potential to determine $\lambda$ with an uncertainty in the 10-20\% range in case LHC provides a luminosity of about $1000 \mathrm{fb}^{-1}$, given that reliable theoretical predictions are at hand.

Leading order corrections to $g g \rightarrow H H$ have been computed quite some time ago in Refs. [3, 4] where the exact dependence on all kinematic variables has been taken into account. Next-to-leading order (NLO) corrections in the effective theory approach where the top quark is integrated out are known since about 15 years [5]. A few months ago also the NNLO corrections became available in this limit $[6]^{1}$ which works very well for single-Higgs boson production. The resummation of soft gluon radiation and dominant $\pi^{2}$ terms have been considered in [7] at next-to-next-to-leading logarithmic order. In Ref. [8] the effect of a finite top quark mass has been considered at NLO. In this contribution the findings of Ref. [8] are summarized.

\section{Technical details and LO result}

The dominant production of Higgs boson pairs is loop-induced and proceeds via gluon fusion. The contributing diagrams can be divided into a triangle contribution, which has a dependence on the triple-Higgs boson coupling and a box contribution where both Higgs bosons directly couple to the top quark loop, see Fig. 1. At NLO both virtual corrections and real emissions have to be considered. In our approach they are incorporated via the optical theorem which connects the total cross section to the imaginary part of the forward scattering amplitude. This has the advantage that, as pointed out in Ref. [9], well-established multi-loop techniques can be applied to compute the Feynman integrals. Sample Feynman diagrams are shown in Fig. 2 where vertical dashed lines
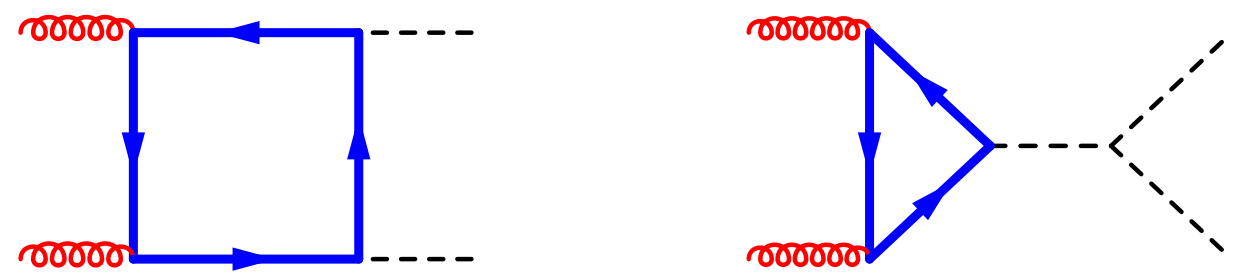

Figure 1: Box and triangle diagrams that contribute to double-Higgs boson production at leading order. Solid lines refer to top quarks and dashed lines refer to Higgs bosons.

\footnotetext{
${ }^{1}$ Note that the effective coupling of gluons to two Higgs bosons is still unknown.
} 

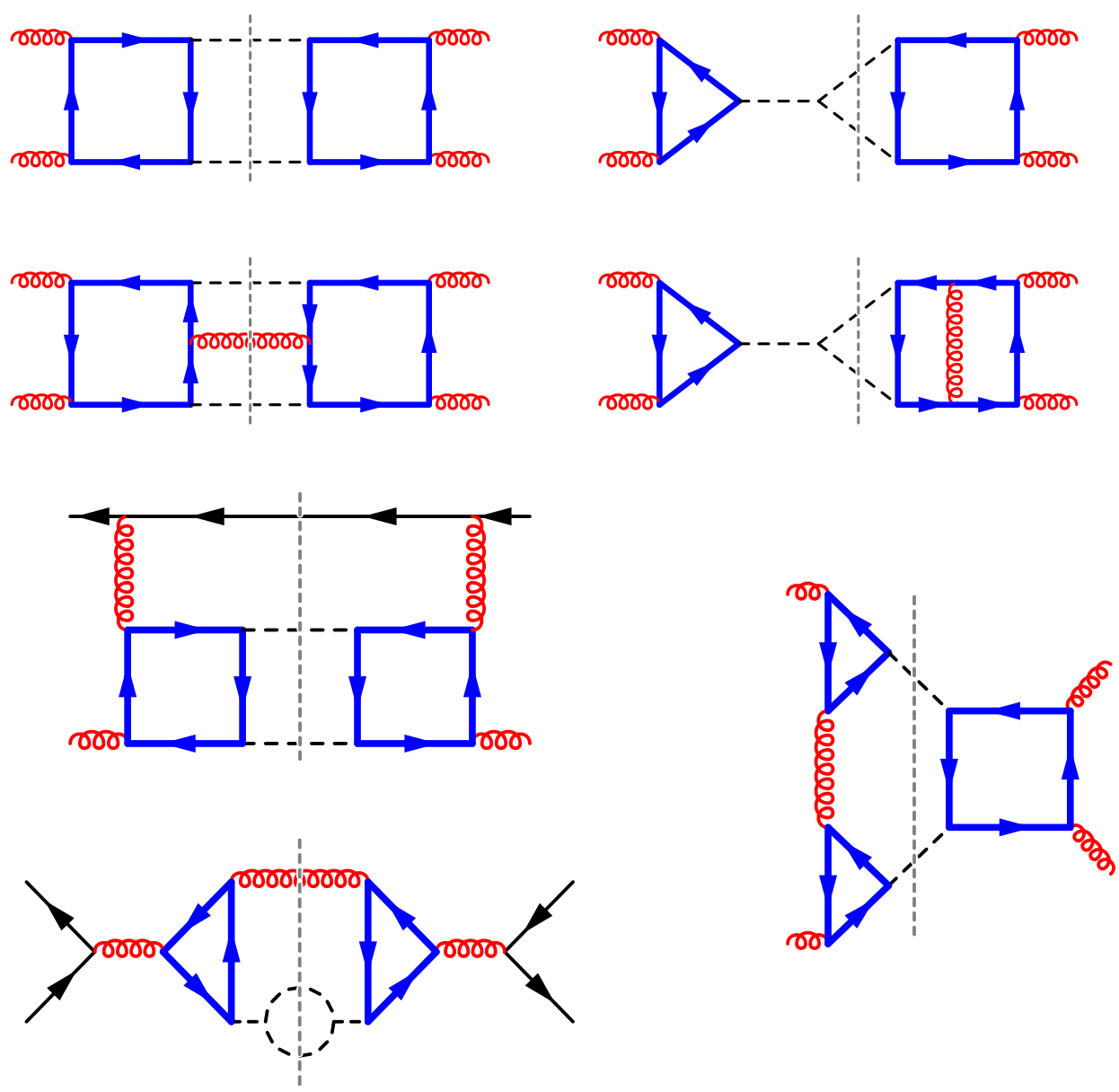

Figure 2: Sample Feynman diagrams for forward scattering kinematics which contribute at LO (top row) and NLO. Dashed vertical lines represent unitarity cuts. Solid lines are top and light quarks, dashed lines are Higgs bosons.

indicate the cuts which lead to the imaginary part. On the right bottom corner of Fig. 2 a Feynman diagrams is shown which contains three closed top quark loops. Such contributions appear for the first time at NLO and contribute to the virtual part. Fig. 2 also contains Feynman diagrams illustrating the quark-gluon and quark-anti-quark initiated channels which appear for the first time at NLO. Note that they are suppressed by at least one order of magnitude and thus in the remainder of this contribution we will restrict the discussion to the gluon-gluon channel.

The LO hadronic result for the Higgs boson pair cross section is shown in Fig. 3 as a function of the invariant Higgs boson mass where curve (b) corresponds to the SM. In order to demonstrate the sensitivity on the triple-Higgs coupling it is switched off in curve (a) which leads to a significantly higher cross section. For comparison we show in curve (c) the result where the box contribution is set to zero. Although this part is much smaller one observes a significant contribution from the interference term. This is particularly true close to threshold where the SM result is significantly smaller than the result from pure box or pure triangle contribution.

The starting point of the NLO calculation are the four-loop diagrams in Fig. 2 which we eval- 


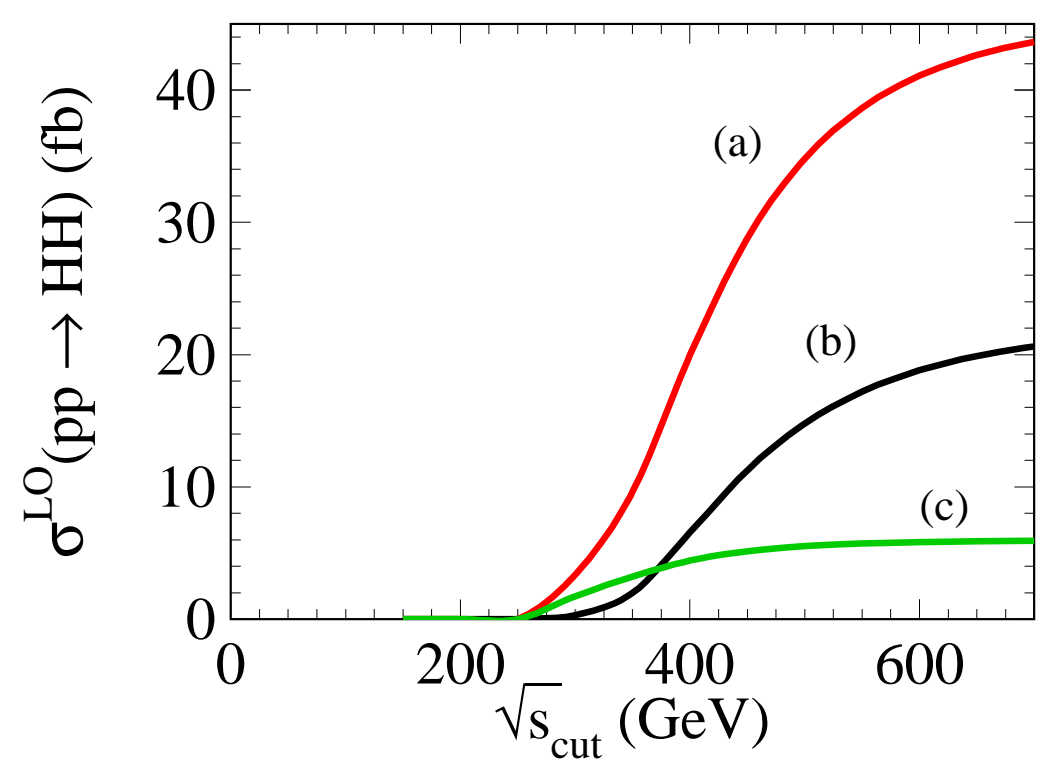

Figure 3: Leading order hadronic cross section for Higgs boson pair production at the $14 \mathrm{TeV} \mathrm{LHC}$ as the function of the upper cut on the Higgs boson pair invariant mass. Curve (b) is the full result; curve (a) is the box contribution; curve (c) is the triangle contribution. The destructive interference between box and triangle contributions is apparent. We use MSTW2008 parton distribution functions [10].

uate in the limit where the top quark mass is the largest scale. In that case the so-called hard-mass procedure (see, e.g., Ref. [11]) can be applied which leads to a factorization of the integrals. To be precise, one obtains products of vacuum integrals up to two loops and one- or two-loop integrals which depend on the Higgs boson mass and the center-of-mass energy $\sqrt{s}$. The former are very well studied in the literature whereas the latter have been considered for the first time in Ref. [8]. We have organized our calculation in such a way that in a first step the integrations associated with the massive vacuum integrals are performed. Afterwards, we have computed integral tables for the remaining one- and two-loop four-point functions with the help of FIRE [12, 13] which led us to four two-loop master integrals. One of them can be expressed as a linear combination of the others as described in Ref. [8]. For the remaining three diagrams an integral representation can be derived which can easily be Taylor-expanded in the parameter $\delta=1-4 m_{H}^{2} / s$. Note that $\delta$ vanishes at threshold. We have computed 100 expansion terms for the partonic cross section and have checked that there is no change in the hadronic cross section in case less terms are taken into account, e.g., only terms up to order $\delta^{50}$. In principle the master integrals could also be evaluated numerically, however, in our approach analytic results for the partonic cross section can be provided.

\section{NLO result}

Using the approach described in the previous section we have computed $\operatorname{six}^{2}$ expansion terms

\footnotetext{
${ }^{2}$ At the time Ref. [8] has been published only five terms were available.
} 


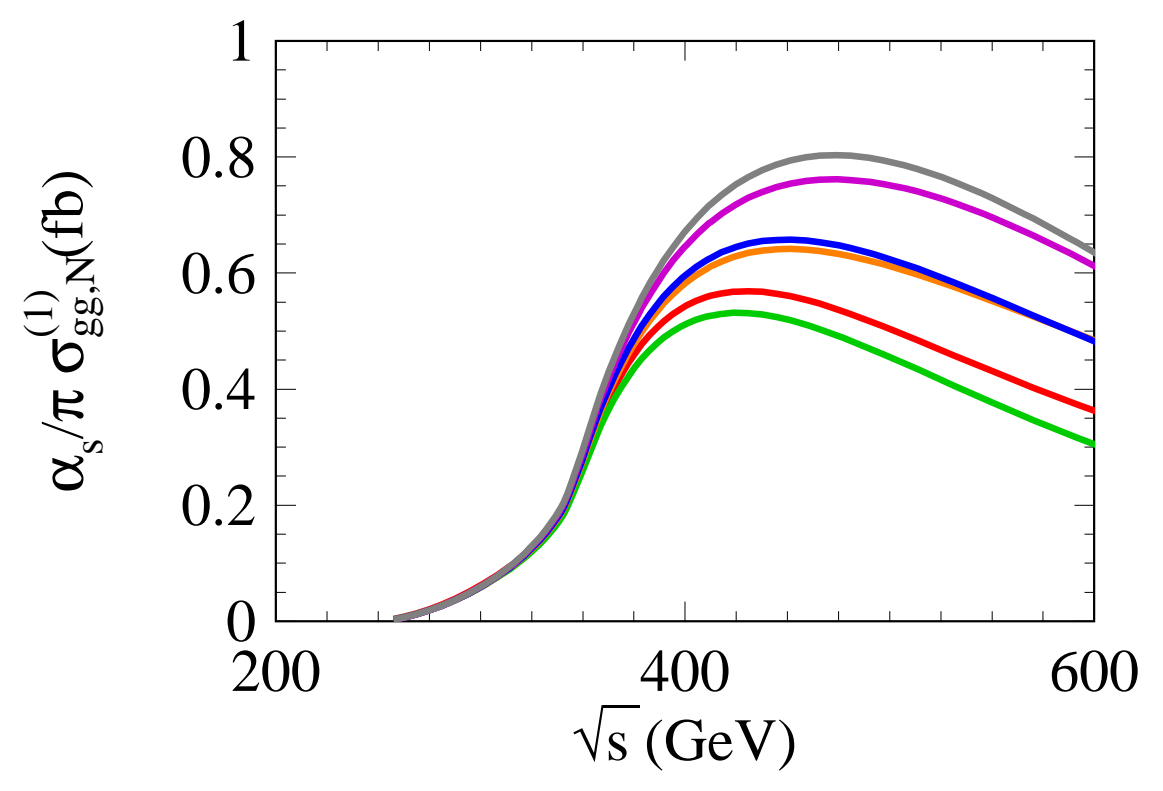

Figure 4: Next-to-leading order contribution to $g g \rightarrow H H$ re-scaled by the exact leading order result where the curves from bottom to top corresponds to $N=1,0,2,3,4,5$. For the Higgs boson and top quark mass we have used $m_{H}=126 \mathrm{GeV}$ and $M_{t}=173.18 \mathrm{GeV}$.

of the partonic cross section in $\rho=m_{H}^{2} / M_{t}^{2}$. On general grounds we expect that such a series shows good convergence properties below the threshold where two real top quarks can be produced, i.e., for $\sqrt{s} \lesssim 346 \mathrm{GeV}$. This is observed in practice. At the same time higher order expansion terms provide sizeable corrections above threshold. In analogy to single-Higgs boson production it is possible to improve the convergence by factoring out the exact leading order cross section and by defining the NLO contribution via

$$
\sigma_{i j, N}^{(1)}=\sigma_{g g, \operatorname{exact}}^{(0)} \Delta_{i j}^{(N)}, \quad \Delta_{i j}^{(N)}=\frac{\sigma_{i j, \exp }^{(1)}}{\sigma_{g g, \exp }^{(0)}}=\frac{\sum_{n=0}^{N} c_{i j, n}^{\mathrm{NLO}} \rho^{n}}{\sum_{n=0}^{N} c_{g g, n}^{\mathrm{LO}} \rho^{n}},
$$

where terms up to $\rho^{N}$ are included in the approximation. Note that the quantity $\Delta_{i j}^{(N)}$ in Eq. (3.1) is a rational function in $\rho$ which is crucial in order to obtain good results.

In Eq. (3.1) the complete LO cross section consisting of triangle and box contributions has been factored out. Alternatively, one could also separate the NLO expression into triangle-triangle, box-box and triangle-box contributions and factor out for each piece the corresponding exact Born cross section. We have implemented also this option and checked that on the practical level there is no difference as compared to the approximation of Eq. (3.1).

In Fig. 4 we show the cross section as a function of the partonic center-of-mass energy $\sqrt{s}$. Reasonable convergence is observed up to $\sqrt{s} \approx 400 \mathrm{GeV}$ but also for higher energies the difference between the curves with $N=0$ and $N=5$ remains bounded. Since the weight of this region is 


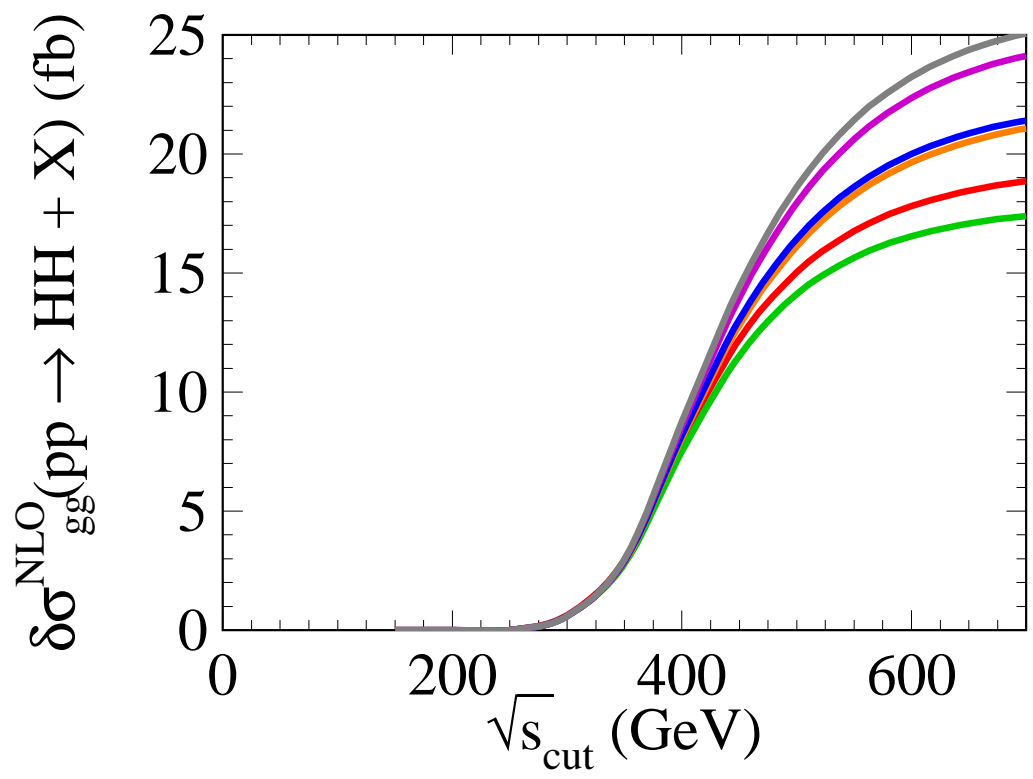

Figure 5: Next-to-leading order contributions for the gluon-gluon channel to the hadronic production cross section of Higgs boson pairs. From bottom to top the curves correspond to $N=1,0,2,3,4,5$.

suppressed in the convolution integral with the parton distribution functions we expect even better results for the hadronic cross section.

Fig. 5 shows the hadronic gluon-induced cross section for $N=0, \ldots, 5$ as a function of $\sqrt{s_{\text {cut }}}$, an upper cut on the partonic center-of-mass energy. At LO $\sqrt{s_{\text {cut }}}$ is equivalent to the two-Higgs boson invariant mass $M_{H H}$. At NLO this is not true any more, however, we use $\sqrt{s_{\text {cut }}}$ as an approximation to the cut on $M_{H H}$. Note that in the limit $\sqrt{s_{\text {cut }}} \rightarrow \infty$ the total cross section is recovered. In particular, for $N=0$ the results of Ref. [5] could be confirmed. ${ }^{3}$

It is interesting to mention that the expansion terms in Fig. 5 group into pairs which contain two successive orders. Note that for $\sqrt{s_{\mathrm{cut}}} \sim 700 \mathrm{GeV}$, the shift in $\delta \sigma_{g g}^{\mathrm{NLO}}$ due to the last computed term in the $1 / M_{t}$ expansion as compared to the prediction including $\rho^{3}$ terms is less than $20 \%$; for lower values of $\sqrt{s_{\text {cut }}}$ the convergence is significantly better. Taking into account that the $K$ factor

\begin{tabular}{l||l|l|l|l|l|l||l}
\multicolumn{1}{c||}{$\sigma^{\mathrm{LO}}(\mathrm{fb})$} & \multicolumn{5}{c||}{$\delta \sigma_{N}^{\mathrm{NLO}}(\mathrm{fb})$} & $\sigma^{\mathrm{NLO}}(\mathrm{fb})$ \\
\hline & $\mathrm{N}=0$ & 1 & 2 & 3 & 4 & 5 & \\
\hline 22.4 & 19.0 & 16.4 & 21.5 & 21.4 & 24.5 & 25.3 & $45.0 \pm 3.9$
\end{tabular}

Table 1: LO and NLO cross sections for double-Higgs boson production including also the quark channels at NLO. No cut on the partonic center-of-mass energy is applied. For $\sigma^{\mathrm{LO}}$ the LO MSTW2008 parton distribution functions [10] have been used and all other results have been obtained using NLO parton distribution functions. We have set the hadronic center-of-mass energy to $14 \mathrm{TeV}$.

\footnotetext{
${ }^{3}$ Note that in Ref. [5] the factorization of the exact LO result has been implemented differently from Ref. [8].
} 


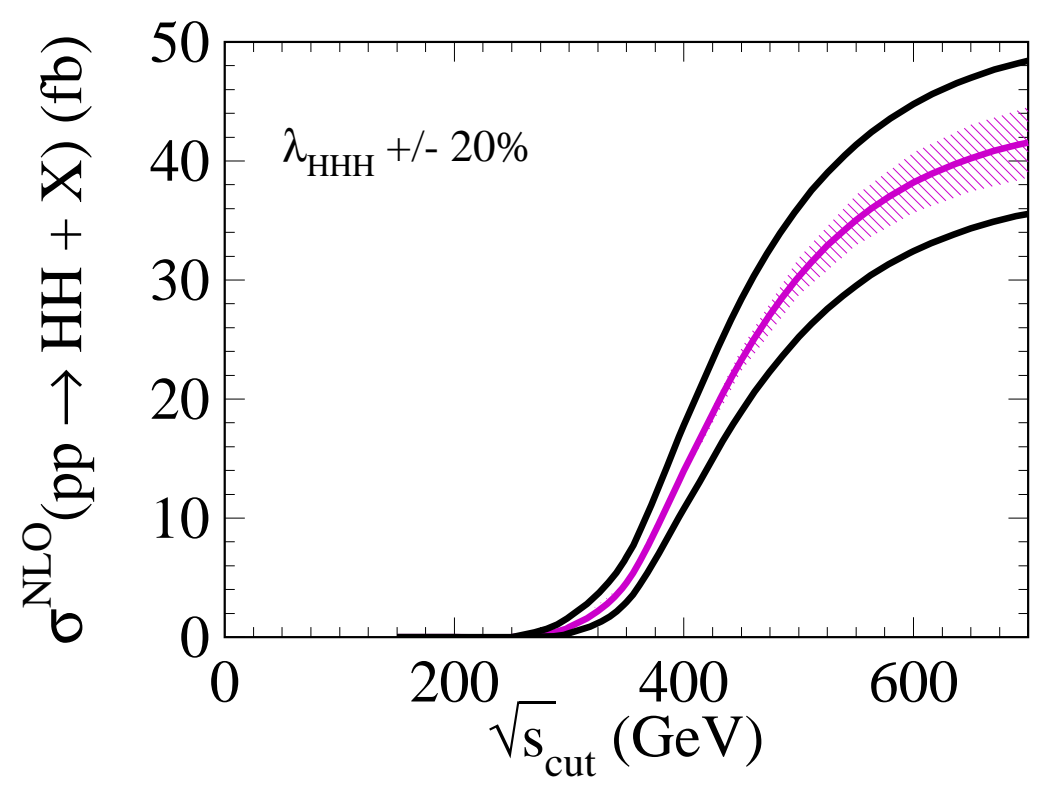

Figure 6: The NLO hadronic cross section at the $14 \mathrm{TeV}$ LHC as a function of $\sqrt{s_{\text {cut }}}$. Two black curves correspond to $\pm 20 \%$ variation in the triple-Higgs boson coupling relative to its SM value.

is close to two these shifts get basically reduced by the same factor when considering the complete NLO prediction.

We refrain to discuss the $K$ factor in detail in this contribution and refer to Ref. [8]. Instead we want to provide results for the total cross section obtained after removing the cut on the partonic center-of-mass energy. In Tab. 1 we summarize the results of $\sigma^{\mathrm{LO}}, \delta \sigma_{N}^{\mathrm{NLO}}$ and $\sigma^{\mathrm{NLO}}=\sigma^{\mathrm{LO}}+$ $\delta \sigma_{5}^{\mathrm{NLO}}$ where the subscript $N$ indicates the used expansion depth in $\rho$ for the partonic cross section. The uncertainty assigned to $\sigma^{\mathrm{NLO}}$ has been obtained by comparing $\delta \sigma_{5}^{\mathrm{NLO}}$ and $\delta \sigma_{3}^{\mathrm{NLO}}$ which is a quite conservative approach.

\section{Concluding remarks}

This contribution summarizes the computation of top quark mass effects to the cross section $\sigma(p p \rightarrow H H)$ at NLO [8]. An expansion for a heavy top quark mass has been applied and six expansion terms have been computed. After factoring out the exact LO result at partonic level one observes a good convergence of the hadronic cross section close to threshold, the energy region most important for the extraction of the triple-Higgs boson coupling. Thus, for the first time it is possible to quantify the importance of finite top quark mass effects. This is illustrated in Fig. 6 where the NLO cross section is shown as a function of $\sqrt{s_{\text {cut }}}$. The (violet) uncertainty band due to uncalculated $1 / M_{t}$ corrections has been obtained by comparing the NLO results including $\rho^{4}$ and $\rho^{3}$ terms which amounts to about $10 \%$. Furthermore, we vary the triple-Higgs boson coupling relative to its SM value by $\pm 20 \%$ which is shown as black curves in Fig. 6 . This leads to the 
conclusion that the current knowledge of $1 / M_{t}$ corrections is sufficient to be sensitive to detect $\mathscr{O}(10 \%)$ deviations in the triple-Higgs boson coupling, relative to its SM value.

Results for the total cross section can be found in Tab. 1. The NLO contributions increase by about $30 \%$ when going from the infinite-top quark mass limit to the result including $\rho^{5}$ terms. Furthermore, we can quantify the uncertainty at NLO due to the lack of exact $M_{t}$ dependence which amounts to about $9 \%$ for the NLO prediction of the total cross section.

\section{Acknowledgments}

The research of J.H, J.G. and M.S. is supported by the Deutsche Forschungsgemeinschaft in the Sonderforschungsbereich Transregio 9 "Computational Particle Physic". The research of K.M. is partially supported by US NSF under grants PHY-1214000. The research of K.M. and J.G. are partially supported by Karlsruhe Institute of Technology through its distinguished researcher fellowship program.

\section{References}

[1] G. Aad et al. [ATLAS Collaboration], Phys. Lett. B716, 1 (2012).

[2] S. Chatrchyan et al., [CMS Collaboration], Phys. Lett. B716, 30 (2012).

[3] E. W. N. Glover and J. J. van der Bij, Nucl. Phys. B 309 (1988) 282.

[4] T. Plehn, M. Spira and P. M. Zerwas, Nucl. Phys. B 479 (1996) 46 [Erratum-ibid. B 531 (1998) 655] [hep-ph/9603205].

[5] S. Dawson, S. Dittmaier and M. Spira, Phys. Rev. D 58, 115012 (1998) [hep-ph/9805244].

[6] D. de Florian and J. Mazzitelli, Phys. Rev. Lett. 111, 201801 (2013) [arXiv:1309.6594 [hep-ph]].

[7] D. Y. Shao, C. S. Li, H. T. Li and J. Wang, arXiv:1301.1245 [hep-ph].

[8] J. Grigo, J. Hoff, K. Melnikov and M. Steinhauser, Nucl. Phys. B 875 (2013) 1 [arXiv:1305.7340 [hep-ph]].

[9] C. Anastasiou and K. Melnikov, Nucl. Phys. B 646, 220 (2002) [hep-ph/0207004].

[10] A. D. Martin, W. J. Stirling, R. S. Thorne and G. Watt, Eur. Phys. J. C 63 (2009) 189 [arXiv:0901.0002 [hep-ph]].

[11] V. A. Smirnov, Analytic tools for Feynman integrals, Springer Tracts Mod. Phys. 250 (2012) 1.

[12] A. V. Smirnov, JHEP 0810 (2008) 107 [arXiv:0807.3243 [hep-ph]].

[13] A. V. Smirnov and V. A. Smirnov, arXiv:1302.5885 [hep-ph]. 